\title{
A Secure and Mutual-Profitable DRM Interoperability Scheme
}

\author{
Sangho Lee \\ Dept. of CSE, POSTECH \\ Pohang, Republic of Korea \\ sangho2@postech.edu
}

\author{
Heejin Park \\ LG Electronics Inc. \\ Seoul, Republic of Korea \\ parkhj84@lge.com
}

\author{
Jong Kim \\ Dept. of CSE, POSTECH \\ Pohang, Republic of Korea \\ jkim@ postech.edu
}

\begin{abstract}
In most cases, the use of digital contents on several devices is blocked by digital rights management (DRM) technology to protect the rights of digital content owners, which is called as the DRM's walled garden strategy. This strategy has raised many legal, economical, and ethical problems. DRM interoperability can complement this strategy. However, there is no agreeable systematic interoperability scheme between various DRM systems. This problem cannot be solved without the cooperation and participation of both DRM technology providers and content providers. Some previous attempts to solve the DRM interoperability problem have suggested that both providers need to open parts of their security properties, without the assurance of a beneficial outcome. They were therefore reticent about participating. In this paper, we propose a secure mutual-profitable DRM interoperability scheme which minimizes disclosure of the security properties of DRM technology providers and content providers while preserving their profits. We use a designated proxy re-encryption scheme to allow the providers to designate a proxy which re-encrypts their digital contents and a neutral format scheme to enable format-independent translations. Moreover, we allow the providers to manage and trace their digital contents, and to request additional fees for interoperability services. We describe detailed protocols and analyze the scheme. We also introduce a prototype implementation.
\end{abstract}

Keywords-Digital Rights Management (DRM), Interoperability, Proxy Re-encryption

\section{INTRODUCTION}

Digital rights management (DRM) was introduced to protect the copyright of digital contents in digital environments. Various DRM technologies are currently available [2], [3]. Most of them take the walled garden strategy [4] to protect the contents they provide and the profit they can get, even though it brings up many legal and ethical problems. One way to complement this strategy is DRM interoperability. Without DRM interoperability, consumers have to repeatedly purchase the same digital contents if they wish to use them on their heterogeneous devices. Consumers frequently criticize content providers because they are generally adopting noninteroperable DRM schemes [5]. On the other hand, a recent survey has shown that many consumers are willing to pay more money for contents with interoperability [6]. Therefore,

The preliminary version of this paper was presented at [1]. This research was supported by the MKE(The Ministry of Knowledge Economy), Korea, under the ITRC(Information Technology Research Center) support program supervised by the NIPA(National IT Industry Promotion Agency)" (NIPA2010-C1090-1031-0009).
DRM interoperability is required to increase activities in the digital market while protecting the digital copyright.

Several researchers have suggested schemes [7], [8], [9], [10] to solve the DRM interoperability problem. According to Koenen et al. [5], there are three possible approaches to interoperability in DRM systems: full format interoperability, connected interoperability, and configuration driven interoperability. Full format interoperability means that every DRM system shares the same security infrastructure, which is feasible by having a standard. However, due to many business reasons, the standard for DRM is still a long way to go. Because full format interoperability is difficult to acquire, an alternative approach which uses a neural format [9] for content translation has been proposed. Devices translate content to a neutral format when exporting it and then convert the received neutral format to their own DRM format while importing it. Some security weaknesses exist in this approach because content translations and license generations are performed by devices. Connected interoperability means that an external trusted entity manages interoperability services [8], [10]. The external trusted entity has to know all the security properties of the DRM technology providers such as encryption methods, content formats, and license formats. However, providers do not want to open their security properties as far as possible. Configuration driven interoperability means that a consumer's device can download heterogeneous DRM components as software to extend its functionality [7]. Because it is softwarebased, it has inherent security weaknesses.

Motivation and Research Goal. To solve the DRM interoperability problem, we need to encourage participation from both DRM technology providers and content providers. Nevertheless, previous studies on the DRM interoperability have considered how to fulfill consumers' needs while largely ignoring how to encourage the participation of DRM technology providers and content providers. Without their participation, DRM interoperability schemes are hard to achieve. Moreover, because the content providers want to make a profit with their digital contents even when it is not used on the original device, they want to trace the usage of their contents. Also, the technology providers are reluctant to disclose their security properties because they do not want to reveal their technology for possible hacking. Previous work, however, would need the 
technology providers to open parts of their security properties and did not consider incorporating tracking and control features within a DRM interoperability scheme.

In this paper, we propose a secure mutual-profitable scheme to address the DRM interoperability problem. The proposed scheme minimizes the disclosure of DRM technology and content providers' security properties by using designated proxy re-encryption and neutral format schemes [9]. The designated proxy re-encryption scheme allows a designated proxy to re-encrypt specific content without revealing the raw content, while the neutral format scheme allows for formatindependent translations. Taban et al. [10] also used a proxy re-encryption scheme [11] for DRM interoperability. Their scheme, however, cannot designate a proxy to perform the re-encryption and also cannot specify the content to be reencrypted. Therefore, if someone were able to obtain a reencryption key from device $A$ to device $B$, he/she could illegally re-encrypt and deliver all contents of the device $A$ to the device $B$. In the proposed scheme, however, if someone obtained a re-encryption key, he/she could only be able to reencrypt specific contents. Therefore, the proposed scheme is more secure than the Taban et al.'s scheme [10]. Moreover, in the proposed scheme, DRM technology and content providers are able to manage and trace DRM interoperability processes, and bill additional fees for DRM interoperability services. This is likely to encourage the providers to actively participate in the scheme to increase DRM interoperability.

Paper Organization. The rest of this paper is organized as follows. In Section II, we introduce preliminaries of this paper. In Section III, we discuss our system model. In Section IV, we describe our scheme and analyze it in Section V. In Section VI, we explain a prototype implementation of our scheme. Finally, we conclude this paper in Section VII.

\section{PRELIMINARIES}

Bilinear Map. A map $e: G_{1} \times G_{1} \rightarrow G_{2}$ is a bilinear map which has the following properties:

- $G_{1}$ and $G_{2}$ are groups of the same prime order $q$.

- For all $a, b \in \mathbb{Z}_{q}$ and $q, h \in G_{1}, e\left(g^{a}, h^{b}\right)=e(g, h)^{a b}$ is efficiently computable.

- The map is non-degenerate, i.e., if $g$ generates $G_{1}$ and $h$ generates $G_{1}$, then $e(g, h)$ generates $G_{2}$.

We set invertible functions $\psi_{1}: \mathbb{Z}_{q} \rightarrow G_{1}$ and $\psi_{2}: \mathbb{Z}_{q} \rightarrow G_{2}$.

Proxy Re-encryption. Proxy re-encryption allows a proxy to transform a ciphertext computed under $A$ 's public key into one that can be opened by $B$ 's secret key without any additional decryption. The temporary unidirectional proxy re-encryption scheme [11] is based on the ElGamal scheme operating over two groups $G_{1}, G_{2}$ of prime order $q$ with a bilinear map $e: G_{1} \times G_{1} \rightarrow G_{2}$. The system parameters are random generators $g \in G_{1}$ and $Z=e(g, g) \in G_{2}$.

Key Generation: User $A$ 's key pair is of the form $s k_{A}=$ $a \in_{R} \mathbb{Z}_{q}^{*}$ and $p k_{A}=g^{a} \in G_{1}$.

Re-Encryption Key Generation: $A$ delegates to $B$ by pub- lishing the re-encryption key $r k_{A \rightarrow B}=g^{b / a} \in G_{1}$, computed from $B$ 's public key.

First-Level Encryption: General public based encryption method is called a first-level encryption. To encrypt a message $m \in G_{2}$ under $p k_{A}$ in such a way that it can only be decrypted by the holder of $s k_{A}, Z^{a k}=e\left(g^{a}, g^{k}\right)$ is computed where $k \in \in_{R} \mathbb{Z}_{q}^{*}$, and $c=\left(Z^{a k}, m Z^{k}\right)$ is the output.

Second-Level Encryption: This encryption is a preliminary encryption for proxy re-encryption. Therefore, second-level encryption should be performed first so that a proxy can perform the re-encryption. To encrypt a message $m \in G_{2}$ under $p k_{A}$ in such a way that it can be decrypted by $A$ and other delegatees, $c=\left(g^{a k} ; m Z^{k}\right)$ is published.

Re-encryption: Anyone can change a second-level ciphertext for $A$ into a first-level ciphertext for $B$ with $r k_{A \rightarrow B}=g^{b / a}$. Using $c_{a}=\left(g^{a k}, m Z^{k}\right), e\left(g^{a k}, g^{b / a}\right)=Z^{b k}$ is computed and $c_{b}=\left(Z^{b k}, m Z^{k}\right)$ is published.

Decryption: To decrypt a first-level ciphertext $c_{a}=(\alpha, \beta)$ with $s k_{A}=a, m=\beta /\left(\alpha^{1 / a}\right)$ is computed and published.

Designated Proxy Re-encryption. Based on the temporary unidirectional proxy re-encryption [11], we propose a designated proxy re-encryption which allows message creators to designate a proxy to perform re-encryption.

Key Generation: A message creator $C$ choose a key pair $s k_{m}=\mu \in_{R} \mathbb{Z}_{q}^{*}$ and $p k_{m}=g^{\mu} \in G_{1}$ for a message $m$.

Re-Encryption Key Generation: $C$ computes a re-encryption key $r k_{\mu \rightarrow b}=g^{b / \mu} \in G_{1}$ which will be used to re-encrypt a message encrypted with a key $\mu$ to a key $b$.

First-Level Encryption: To encrypt a message $m \in G_{2}$ under $p k_{A}=g^{a}$ in such a way that it can only be decrypted by the holder of $s k_{A}=a, c_{A}=\left(Z^{a k}=e\left(g^{a}, g\right)^{k}, m Z^{k}\right)$ is computed and published where $k \in_{R} \mathbb{Z}_{q}^{*}$.

Second-Level Encryption: To encrypt a message $m \in G_{2}$ under $p k_{m}$ in such a way that it can only be re-encrypted by the holder of $s k_{\Pi}=\pi \in \mathbb{Z}_{q}^{*}, Z^{\pi k}=e\left(g^{\pi}, g^{k}\right)$ is computed, and $c=\left(g^{\mu k}, m Z^{\pi k}\right)$ is published.

Re-encryption: Only $\Pi$ who has $s k_{\Pi}=\pi$ can change a second-level ciphertext of a message $m$ into a first-level ciphertext for $B$ with $r k_{\mu \rightarrow b}$. From $c=\left(g^{\mu k}, m Z^{\pi k}\right)$, $Z^{b \pi k}=e\left(g^{\mu k}, g^{b / \mu}\right)^{\pi}$ is computed and $c_{B}=\left(Z^{b \pi k}, m Z^{\pi k}\right)$ is published.

Decryption: To decrypt a first-level ciphertext $c_{B}=(\alpha, \beta)$ with $s k_{B}=b, m=\beta /\left(\alpha^{1 / b}\right)$ is computed and published.

\section{System Model AND REQUiREMENTS}

In this section, we introduce a system model and the requirements of our scheme. The rest of this paper uses notations shown in Table I.

\section{A. System Model}

Our system comprised of six kinds of entities: DRM server, content provider, DRM interoperability server, DRM interoperability agent, device, and billing server (see Fig. 1). 
TABLE I

NOTATIONS

\begin{tabular}{|c|c|}
\hline Symbol & Meaning \\
\hline$I D_{m}$ & Identifier of content $m$ \\
\hline$C_{m}$ & Normal format of content $m$ \\
\hline$I C_{m}$ & Interoperable format of content $m$ \\
\hline lic & License \\
\hline$r k_{\mu \rightarrow \alpha}$ & $\begin{array}{l}\text { Re-encryption key to re-encrypt a message encrypted } \\
\text { with a key } \mu \text { to a key } \alpha\end{array}$ \\
\hline$E_{1}(\mu ; m)$ & First-level encryption on a message $m$ with a key $\mu$ \\
\hline$E_{2}(\pi, \mu ; m)$ & $\begin{array}{l}\text { Second-level encryption on a message } m \text { with } \\
\text { a key } \mu \text { designated to a holder of a key } \pi\end{array}$ \\
\hline$S E\left(K_{m} ; m\right)$ & $\begin{array}{l}\text { Symmetric key encryption on a message } m \text { with } \\
\text { a key } K_{m}\end{array}$ \\
\hline
\end{tabular}

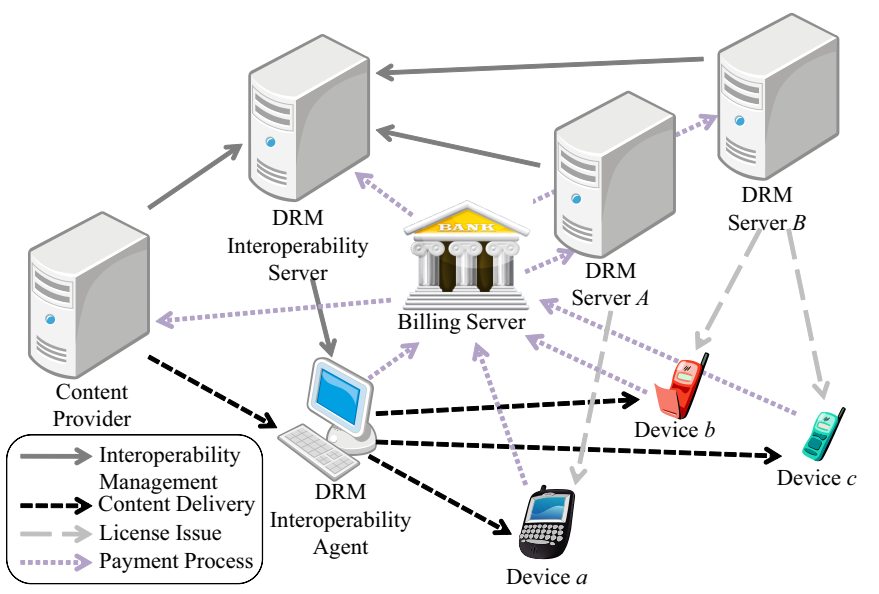

Fig. 1. System model

DRM Server $(D S)$ : DRM server is a DRM technology provider entity which manages DRM devices and issues licenses for contents.

Content Provider $(C P)$ : Content provider is an entity which owns contents and publishes them in a secure format. In our model, it can publish contents in two formats: a general format with content $m$ for a device which has a secret key $\alpha$,

$$
C_{m}=\left(\text { metadata }, E_{1}\left(\alpha ; K_{m}\right), S E\left(K_{m} ; m\right)\right),
$$

or an interoperable format with $m$ for $D I A$ which has a secret key $\pi$,

$$
I C_{m}=\left(\text { metadata }, E_{2}\left(\pi, \mu ; K_{m}\right), S E\left(K_{m} ; m\right)\right) .
$$

DRM Interoperability Server (DIS): DRM interoperability server is the entity which manages overall DRM interoperability processes. When an interoperability service is initiated, it obtains a re-encryption key from $C P$ and $D S$, and delivers it to a DRM interoperability agent $(D I A)$.

DRM Interoperability Agent ( $D I A$ ): DRM interoperability agent is an entity which translates $I C_{m}$ to $C_{m}$. It converts the encrypted key $K_{m}$ of $I C_{m}$ without disclosure when a device requests $I C_{m}$. To do this, it requests a re-encryption key to $D I S$ and performs re-encryption.
Device $(D)$ : Device is an entity which is used by a consumer. A consumer can request $C_{m}$ from a $D I A$ via his/her device. The device can convert $C_{m}$ to its own format to use it.

Billing Server $(B S)$ : Billing server is an entity which manages the overall billing processes.

\section{B. Requirements}

Based on previous research [5], [12], [13], [14], [15], we introduce the following requirements for DRM interoperability schemes.

Persistent Protection: A DRM interoperability scheme has to guarantee the persistent protection of DRM contents. It means that irrespective of translation of DRM contents, the constraints that are imposed by DRM servers have to be enforced.

Security: A DRM interoperability scheme has to guarantee its security against several security attacks such as impersonation and replay attacks. Also, it needs to be protected against bogus $D I A$ s.

Tracking the Translation of DRM Contents: A DRM interoperability scheme has to provide an ability to track the translation of DRM contents to prevent illegal translations by illegitimate entities.

Changing Rights during Translations: Because the policies of DRM technology providers and the functionality of their devices are different, it is difficult to apply the same license model to various DRM systems. Therefore, a DRM interoperability scheme has to allow changes of rights during translations.

Guaranteeing Content Originality: Content originality means that even if contents are converted, their ownership has to be linked with their original DRM server. When contents are re-distributed to other devices, a DRM interoperability scheme has to be able to guarantee the contents originality, i.e., the original DRM server has to be able to manage and trace the re-distribution process.

\section{Assumptions}

We have made the following assumptions for our scheme:

- Each entity, $D S, C P, D I S, D I A, D$, and $B S$, has a certificate for authentication and revocation.

- Entities create a secure channel using their certificates for secure communications between them, e.g., Transport Layer Security (TLS) [16].

- We only consider conceptual payment procedures. Other ideas such as a micro-payment scheme [17] may be integrated into our scheme for practical payment purposes.

\section{Proposed Scheme}

The DRM interoperability problem cannot be solved without the participation of the DRM technology providers and content providers. To encourage the participation of both providers, we have to minimize disclosure of their security 
properties and assure it is of benefit to them. To minimize the disclosure of security properties, we use designated proxy reencryption and neutral format schemes. The designated proxy re-encryption scheme ensures that only a designated $D I A$ can re-encrypt $I C \mathrm{~s}$, while the neutral format scheme eliminates the need for the providers to open their security properties. Also, to ensure this approach is of benefit to them, we propose two protocols to manage the DRM interoperability processes. The first protocol is an acquisition protocol to acquire the $I C$. In this protocol, a consumer purchases $I C_{m}$ from $C P$ via his/her $D I A$ and then stores them on his/her $D I A$. The second protocol is a transmission protocol to deliver the $I C_{m}$ stored on a $D I A$ to a device $A$. To deliver the $I C_{m}$ to $A$, $D I A$ has to re-encrypt the $I C_{m}$ to $C_{m}$ with a re-encryption key which is created by the $D I S, C P$, and $D S_{A}$. Then, to use the $C_{m}, A$ has to purchase a corresponding license from the $D S_{A}$. This payment is distributed to the $D I S, C P$, and $D S_{A}$ to ensure that the DRM technology providers and content providers benefit from in the DRM interoperability process.

\section{A. Acquisition Protocol}

In the acquisition protocol, a consumer buys $I C_{m}$ from the $C P$ through his/her $D I A$. Along with content $m$ 's information and payment information, the DIA sends its own information which includes its public key $g^{\pi}$ and its server $D I S$ 's information to the $C P$. The $C P$ verifies the payment information and the information from the $D I A$ and $D I S$. Then, to create $I C_{m}$ for the $D I A, C P$ encrypts $m$ with a symmetric secret key $K_{m}$, and then performs two-level encryption on $K_{m}$ with an asymmetric secret key $\mu, D I A$ 's public key $g^{\pi}$, and a randomly selected asymmetric secret key $k_{1}$ as $E_{2}\left(\pi, \mu ; K_{m}\right)=\left(g^{\mu k_{1}}, \psi_{2}\left(K_{m}\right) \cdot Z^{\pi k_{1}}\right)$. The created $I C_{m}=$ (metadata, $\left.E_{2}\left(\pi, \mu ; K_{m}\right), S E\left(K_{m} ; m\right)\right)$ is then stored on the DIA for further transmissions (see Fig. 2a).

\section{B. Transmission Protocol}

Assume that a consumer wants to play $m$ which is stored on a $D I A$ with his/her device $A$. $A$ sends a request for $m$ to the $D I A$ along with its information and its server $D S_{A}$ 's information. If the information of $m, A$, and $D S_{A}$ is valid, $D I A$ requests a re-encryption key $r k_{\mu \rightarrow \alpha}$ from the $D I S$ along with its information and information about $m, A, D S_{A}$, and $C P$. The $D I S$ checks the validity of the information about the $D I A$ and $C P$, and then sends a request for $g^{\alpha}$ to $D S_{A}$ along with its information and information about $m, A$, and $C P$. When the received information is valid, $D S_{A}$ randomly creates an asymmetric secret key $\alpha$ and sends $g^{\alpha}$ to $D I S$. $D S_{A}$ stores information of $m, A$, and $\alpha$ to issue licenses later. The DIS sends $g^{\alpha}$ to the $C P$ along with its information and information about $m$ and $D S_{A}$. The $C P$ verifies the received information and then returns $r k_{\mu \rightarrow \alpha}=g^{\alpha / \mu}$ to the DIS.DIS sends $r k_{\mu \rightarrow \alpha}$ to DIA. Then, DIA re-encrypts $E_{2}\left(\pi, \mu ; K_{m}\right)$ as $E_{1}\left(\alpha ; K_{m}\right)=\left(Z^{\alpha \pi k_{1}}, \psi_{2}\left(K_{m}\right) \cdot Z^{\pi k_{1}}\right)$ and sends $C_{m}=$ (metadata, $\left.E_{1}\left(\alpha ; K_{m}\right), S E\left(K_{m} ; m\right)\right)$ to $A$. To decrypt $C_{m}$, $A$ sends a request for the secret key $\alpha$ to $D S_{A}$ along with information about itself and $m$. The $D S_{A}$ verifies the received

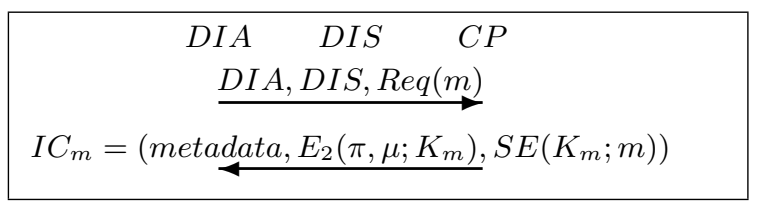

(a) Acquisition protocol

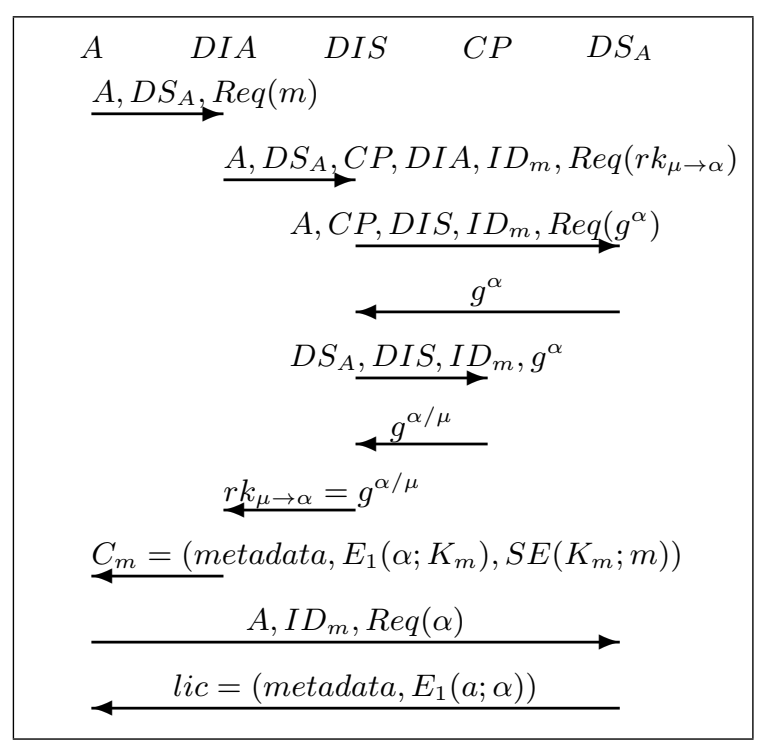

(b) Transmission protocol

Fig. 2. Flow of acquisition and transmission protocols

information and computes $E_{1}(a ; \alpha)=\left(Z^{a k_{2}}, \psi_{2}(\alpha) \cdot Z^{a k_{2}}\right)$. Then, the $D S_{A}$ sends a license lic $=\left(\right.$ metadata, $\left.E_{1}(a ; \alpha)\right)$ to $A$ (see Fig. 2b).

\section{Content Usage}

Because the $C_{m}$ that was translated from the $I C_{m}$ includes a neutral format [9] of content $m$, a device $A$ has to decrypt the $C_{m}$ with a secret key $\alpha$ in the $l i c$, and then transforms $m$ to its own format to use it. To avoid repeated transforming, a device can store the transformed $m$ in a secure storage if it is available, e.g., Trusted Platform Module (TPM) [18].

\section{Billing Scenario}

To encourage the participation of the DRM technology providers and content providers in solving the DRM interoperability problem, we have to ensure that this scheme is of benefit to them. We introduce two kinds of payment: $P_{o}$ and $P_{t} . P_{o}$ is the price of content including an interoperability approval fee, and $P_{t}$ is the price of transmission. We can classify the billing scenario into two cases: on-demand payment and pre-payment.

On-demand Payment: In the acquisition protocol, a consumer pays $P_{o}$ to the $B S$ when purchasing $I C$. Then, in the transmission protocol, the $D I A$ requests a re-encryption key from the $D I S$. Before it gives the re-encryption key to the $D I A$, the $D I S$ asks the content is interoperable with the $D S_{A}$ and whether $P_{t}$ has been paid to the $B S$. After verifying the payment of $P_{t}$, the $B S$ generates a random 
number $R=R_{1} \| R_{2}$ and then creates the payment data:

PaymentData $=\left(H(R)\|D I A\| D I S\|C P\| D S_{A} \| I D_{m}\right)$.

The payment data is stored in the $B S$ as evidence for $P_{t}$. The $B S$ sends this random number $R=R_{1} \| R_{2}$ to $D I S$. Next, the $D I S$ transfers $R_{1}$ to the $C P$ and $R_{2}$ to the $D S_{A}$ with reencryption key request messages. The subsequent billing scenario starts after the device $A$ obtains a corresponding license from the $D S_{A}$. The $D S_{A}$, which issues a new license, requests its profit from the $B S$. At this time, $C P$ and $D S_{A}$ transmit $R_{1}$ and $R_{2}$ as evidence of completed content transmission to the $B S$. Then, the $B S$ compares a hash value of $R=R_{1} \| R_{2}$ with the payment data. If they are same, then $B S$ pays $P_{t}$ to $C P, D S_{A}$, and $D I S$ as the ratio of $p, q$, and $r(p+q+r=1)$.

Pre-payment: The payment certificate is purchased in advance for proof of payment in content transmissions. Initially, consumers purchase the following payment certificate from the $B S$ through the $D I A$.

$$
\text { PaymentCert }=(H(R)\|D I A\| \# \text { transmissions })
$$

When the $D I A$ requests a re-encryption key from the $D I S$, the $B S$ examines the $D I A$ 's payment certificate. At this stage, the $B S$ compares the $D I A$ 's payment certificate with the certificate it stores. If they are same, it reduces the number of transmissions by 1 and then creates payment data for this transmission. The remainder is the same as in the on-demand payment situation.

\section{AnAlysis}

We analyze our scheme according to the requirements in Section III.

Persistent Protection: In our scheme, when contents are translated and delivered to a device, the content encryption key is re-encrypted with a secret key $\alpha$ which is selected by the $D S$ of that device. Thus, each device has to obtain a corresponding license from its $D S$ to know $\alpha$. Therefore, persistent protection is guaranteed.

Security: We analyze the security of our scheme. First, no attacker can impersonate a legal entity because each entity has a certificate for authentication. Second, the $D I A$ cannot obtain the content encryption key of $I C$ because that key is encrypted with a secret key $\mu$ which is selected by $C P$. Also, that key will not be revealed during re-encryption. Third, a device cannot obtain the raw content of $I C$ until it receives a corresponding license because the content encryption key of the $I C$ is encrypted with a secret key $\alpha$ which is selected by its $D S$. Fourth, a bogus $D I A$ cannot give translated $I C$ to other devices because it is encrypted with a secret key $\alpha$ which is selected by $D S$. Devices of other $D S$ es cannot obtain $\alpha$. Also, other devices of the same $D S$ cannot obtain $\alpha$ because that $D S$ will not give licenses to devices that did not purchase that $I C$.

Tracking the Translation of DRM Contents: In our scheme, $D I A$ has to receive a re-encryption key from $C P$ and $D S$ with every transmission. Otherwise, it cannot re-encrypt $I C$.
TABLE II

COMPARISON ON THE RUNNING TIME BETWEEN FUNCTIONS OF PRE2 AND DPRE (FOR A 160-Bit GRoup With AN INTEL PENTIUM $43.0 \mathrm{GHz}$ CPU)

\begin{tabular}{|c|c|c|c|}
\hline \multirow[b]{2}{*}{ Functions } & \multicolumn{2}{|c|}{ Running time (ms) } & \multirow[b]{2}{*}{ Overhead (\%) } \\
\hline & PRE2 & DPRE & \\
\hline gen_params() & 307.9 & - & - \\
\hline keygen() & 61.92 & - & - \\
\hline level1_encrypt() & 9.73 & - & - \\
\hline level2_encrypt() & 18.82 & 53.30 & 283.2 \\
\hline delegate() & 33.57 & - & - \\
\hline reencrypt () & 32.09 & 47.68 & 148.6 \\
\hline decrypt() & 9.52 & - & - \\
\hline
\end{tabular}

By using this, the $C P$ can trace translations of its contents.

Changing Rights during Translations: In our scheme, the rights of the re-distributed contents can be changed because $D S$ issues new licenses at the end of the translations. Therefore, our scheme supports changes of rights during translations.

Guaranteeing Content Originality: In our scheme, only $C P$ can create second-level encrypted contents $I C$. As an $I C$ is translated by $D I A$, it is changed to a first-level encrypted form which cannot be translated to other forms. Therefore, the content originality is guaranteed because only the $C P$ can allow re-distribution of its contents.

\section{PRototype Implementation}

We implement a prototype using the proxy re-cryptography library [19] in a Linux system. The proxy re-cryptography library uses the Multiprecision Integer and Rational Arithmetic $\mathrm{C} / \mathrm{C}++$ Library (MIRACL) [20]. It has two algorithms, PRE1 and PRE2, of Ateniese et al.'s [11]. The PRE2 algorithm is the algorithm which was introduced in Section II; thus, we implement the designated proxy re-encryption (DPRE) algorithm by modifying it. The DPRE algorithm is comprised of seven functions:

- gen_params(): generate domain parameters

- keygen(): generate a public/private key pair

- level1_encrypt(): perform first-level encryption

- level2_encrypt(): perform second-level encryption

- delegate(): generate a re-encryption key

- reencrypt(): re-encrypt a second-level encrypted message

- decrypt(): decrypt an encrypted message

The gen_params(), keygen(), level1_encrypt(), delegate(), and decrypt() functions of the DPRE are same for each of the PRE2. The level2_encrypt() and reencrypt() functions are modified to use the public/private key pair of the $D I A$. The level2_encrypt() function of DPRE is about 2.8 times slower than the PRE2 because of the additional bilinear map operation and the reencrypt() function of DPRE is about 1.5 times slower than the PRE2 because of the additional exponentiation (see Table II). This overhead is not a big problem because these two functions are used by servers.

We also implement four simple programs that represent the entities of our system model: DPRE_CP for $C P$, DPRE_DS 


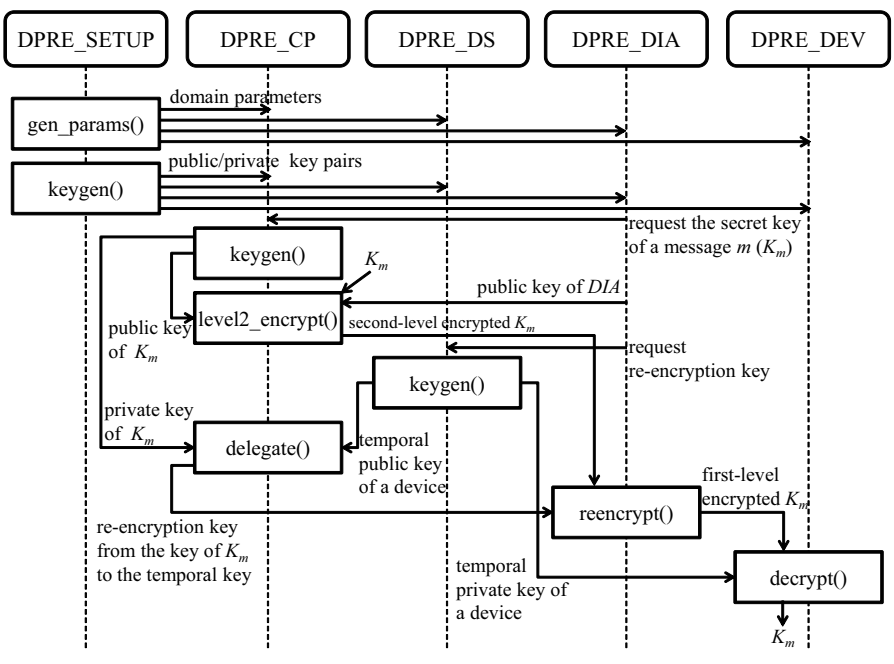

Fig. 3. Functional entities and their interactions

for $D S$, DPRE_DIA for $D I A$ and $D I S$, and DPRE_DEV for $D$. In addition, we implement DPRE_SETUP which generates domain parameters and public/private key pairs of the above programs. The interactions between these programs are as follows (see Fig. 3).

1) The DPRE_SETUP generates domain parameters and public/private key pairs of each program. It then sends them to each program.

2) When the DPRE_DIA requests the secret key of a message $m\left(K_{m}\right)$, the DPRE_CP generates a public/private key pair for $K_{m}$, and then performs second-level encryption on $K_{m}$ with the public keys of $K_{m}$ and DPRE_DIA. It sends the result to the DPRE_DIA.

3) After the DPRE_DIA receives the second-level encrypted $K_{m}$, it requests a re-encryption key to the DPRE_DS. The DPRE_DS generates a temporal public/private key pair of a device, and then sends the temporal public key to the DPRE_CP and the temporal private key to the DPRE_DEV. The DPRE_CP generates the re-encryption key using the temporal public key and the private key of $K_{m}$, and then sends it to the DPRE_DIA.

4) After the DPRE_DIA receives the re-encryption key, it performs re-encryption on the second-level encrypted $K_{m}$ to generate the first-level encrypted $K_{m}$. It sends the result to the DPRE_DEV.

5) The DPRE_DEV decrypts the first-level encrypted $K_{m}$ with the temporal private key.

\section{CONCLUSION}

In this paper, we proposed a secure mutual-profitable interoperable DRM scheme which guarantees the needs and requirements of both providers and consumers. Our scheme uses designated proxy re-encryption and neutral format schemes to minimize the disclosure of security properties of DRM technology providers and content providers, and suggests a billing scenario to encourage the participation of both providers to solve the DRM interoperability problem. Therefore, our scheme meets the needs of consumers and providers, and allows for effective interoperable DRM systems.

\section{ACKNOWLEDGEMENT}

We would like to thank Seungkwang Lee for his help in the implementation.

\section{REFERENCES}

[1] H. Park, S. Lee, and J. Kim, "Rights-preserving interoperability protocol in DRM," in Proceedings of Conference on Information Security and Cryptology - Winter 2008 (CISC-W08), 2008, pp. 141-144, text in Korean.

[2] S. Michiels, W. Joosen, E. Truyen, and K. Verslype, "Digital rights management-a survey of existing technologies," Department of Computer Science, Katholieke Universiteit Leuven, Tech. Rep., November 2005.

[3] B. Rosenblatt, B. Trippe, and S. Mooney, Digital Rights ManagementBusiness and Technology. New York: M\&T Books, 2002.

[4] N. W. Netanel, "Temptations of the walled garden: Digital rights management and mobile phone carriers," Journal on Telecommunications and High Technology Law, vol. 6, 2007.

[5] R. H. Koenen, J. Lacy, M. Mackay, and S. Mitchell, "The long march to interoperable digital rights management," Proceedings of IEEE, vol. 92, no. 6, pp. 883-897, June 2004.

[6] N. Dufft, A. Stiehler, D. Vogeley, and T. Wichmann, "Digital music usage and DRM-results from an european consumer survey," May 2005, http://www.indicare.org/survey.

[7] ISO/IEC 14496-13, "Information technology: Generic coding of moving pictures and associated audio information, part 2: IPMP on MPEG-2 systems," 2002.

[8] D. W. Kravitz and T. S. Messerges, "Achieving media portability through local content translation and end-to-end rights management," in Proceedings of the 5th ACM workshop on Digital Rights Management, 2005, pp. 27-36.

[9] D.-W. Nam, J.-S. Lee, and J.-H. Kim, "Interlock system for DRM interoperability of streaming contents," in Proceedings of IEEE International Symposium on Consumer Electronics 2007 (ISCE 2007), 2007.

[10] G. Taban, A. A. Cárdenas, and V. D. Gligor, "Towards a secure and interoperable DRM architecture," in Proceedings of the 6th ACM workshop on Digital Rights Management, 2006, pp. 69-78.

[11] G. Ateniese, K. Fu, M. Green, and S. Hohenberger, "Improved proxy re-encryption schemes with applications to secure distributed storage," ACM Transactions on Information and System Security (TISSEC), vol. 9, no. 1 , pp. 1-30, 2006.

[12] A. Arnab and A. Hutchison, "Digital rights management-an overview of current challenges and solutions," in Proceedings of Information Security South Africa (ISSA), 2004.

[13] F. Bartolini, V. Cappellini, A. Piva, and A. Fringuelli, "Electronic copyright management systems: Requirements, players and technologies," in Proceedings of the 10th International Workshop on Database and Expert System Applications, 1999, pp. 896-898.

[14] D. Mulligan, J. Han, and A. J. Burstein, "How DRM-based content delivery system disrupt expectations of "personal use"," in Proceedings of the 3rd ACM workshop on Digital Rights Management, 2003, pp. $77-89$.

[15] J. Park, R. Sandhu, and J. Schifalacqua, "Security architectures for controlled digital information dissemination," in Proceedings of the 16th Annual Computer Security Applications Conference (ACSAC'00), 2000, pp. 224-233.

[16] T. Dierks and E. Rescorla, "The Transport Layer Security (TLS) Protocol Version 1.2," RFC 5246 (Proposed Standard), Internet Engineering Task Force, Aug. 2008. [Online]. Available: http://www.ietf.org/rfc/rfc5246.txt

[17] M.-S. Hwang, I.-C. Lin, and L.-H. Li, "Simple micro-payment scheme," Journal of Systems and Software, vol. 55, no. 3, pp. 221-229, January 2001.

[18] Trusted Computing Group, http://www.trustedcomputinggroup.org/.

[19] The JHU-MIT Proxy Re-cryptography Library, http://spar.isi.jhu.edu/prl/.

[20] Multiprecision Integer and Rational Arithmetic C/C++ Library (MIRACL), http://www.shamus.ie/. 